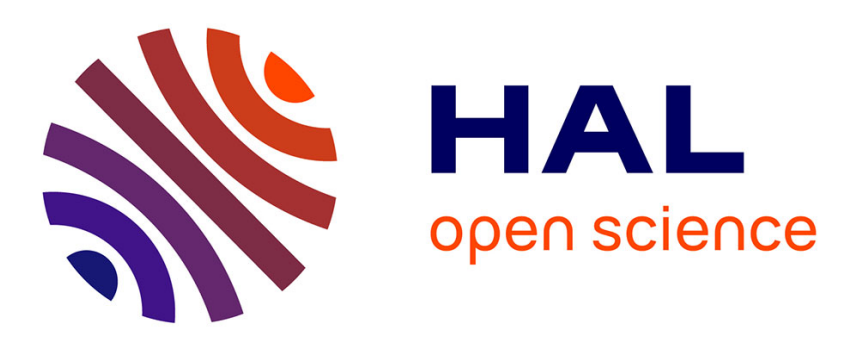

\title{
PCB sssembly scheduling for collect-and-place machines using genetic algorithms
}

Hans-Otto Guenther, Ihsan Onur Yimaz, Osman Kulak

\section{To cite this version:}

Hans-Otto Guenther, Ihsan Onur Yimaz, Osman Kulak. PCB sssembly scheduling for collect-andplace machines using genetic algorithms. International Journal of Production Research, 2007, 45 (17), pp.3949-3969. 10.1080/00207540600791608 . hal-00512922

\section{HAL Id: hal-00512922 \\ https://hal.science/hal-00512922}

Submitted on 1 Sep 2010

HAL is a multi-disciplinary open access archive for the deposit and dissemination of scientific research documents, whether they are published or not. The documents may come from teaching and research institutions in France or abroad, or from public or private research centers.
L'archive ouverte pluridisciplinaire HAL, est destinée au dépôt et à la diffusion de documents scientifiques de niveau recherche, publiés ou non, émanant des établissements d'enseignement et de recherche français ou étrangers, des laboratoires publics ou privés. 


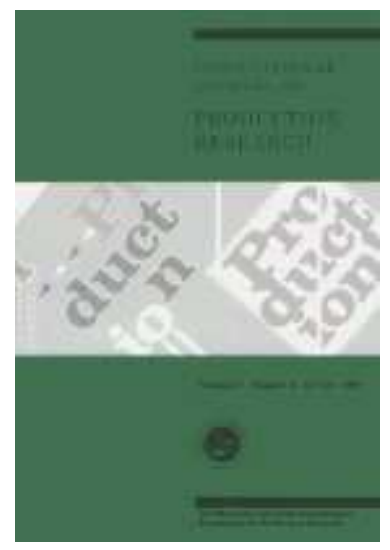

\section{PCB sssembly scheduling for collect-and-place machines using genetic algorithms}

\begin{tabular}{|r|l|}
\hline Journal: & International Journal of Production Research \\
\hline Manuscript ID: & TPRS-2005-IJPR-0291.R1 \\
\hline Manuscript Type: & Original Manuscript \\
\hline $\begin{array}{r}\text { Date Submitted by the } \\
\text { Author: }\end{array}$ & 23-Apr-2006 \\
\hline Complete List of Authors: & $\begin{array}{l}\text { Guenther, Hans-Otto; TU Berlin, Production Management } \\
\text { Yimaz, Ihsan; TU Berlin, Production Management } \\
\text { Kulak, Osman; Pamukkale University, Dept. of Industrial } \\
\text { Engineering }\end{array}$ \\
\hline Keywords: & GENETIC ALGORITHMS, ELECTRONICS ASSEMBLY \\
\hline Keywords (user): & \\
\hline
\end{tabular}

\section{S) Scholarone}

Manuscript Central 


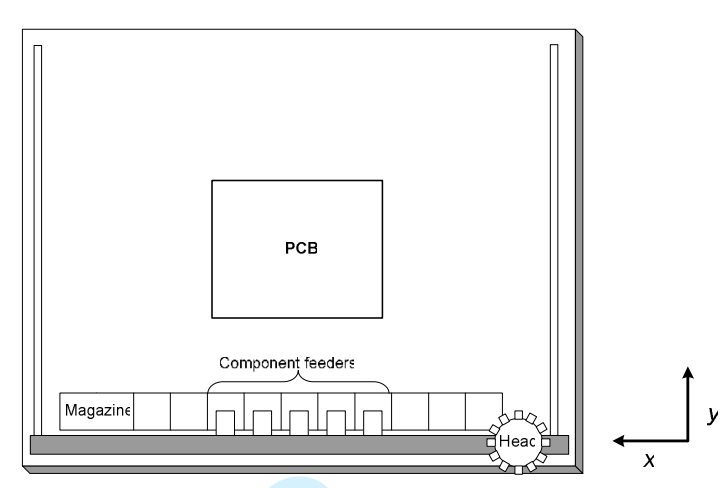

(a) single-gantry machine

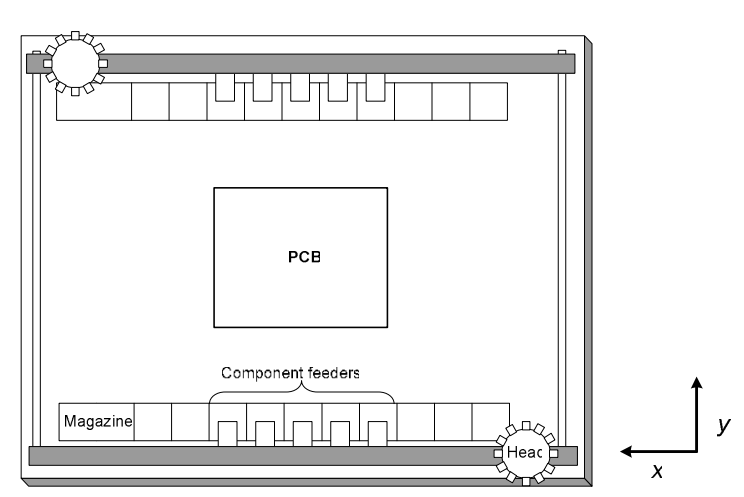

(b) dual-gantry machine

Figure 1. Single- and dual-gantry collect-and-place machines 
Figure 2. Partial-link chromosome structure ( $a_{i}$ : component type, $i=1,2,3, \ldots, m ; b_{j}$ : sequence of placement operations, $j=1,2,3, \ldots, n$ ) 


1
2
3
4
5
6
7
8
9
10
11
12
13
14
15
16
17
18
19
20
21
22
23
24
25
26
27
28
29
30
31
32
33
34
35
36
37
38
39
40
41
42
43
40
45
49
50
51
52
53
55
50

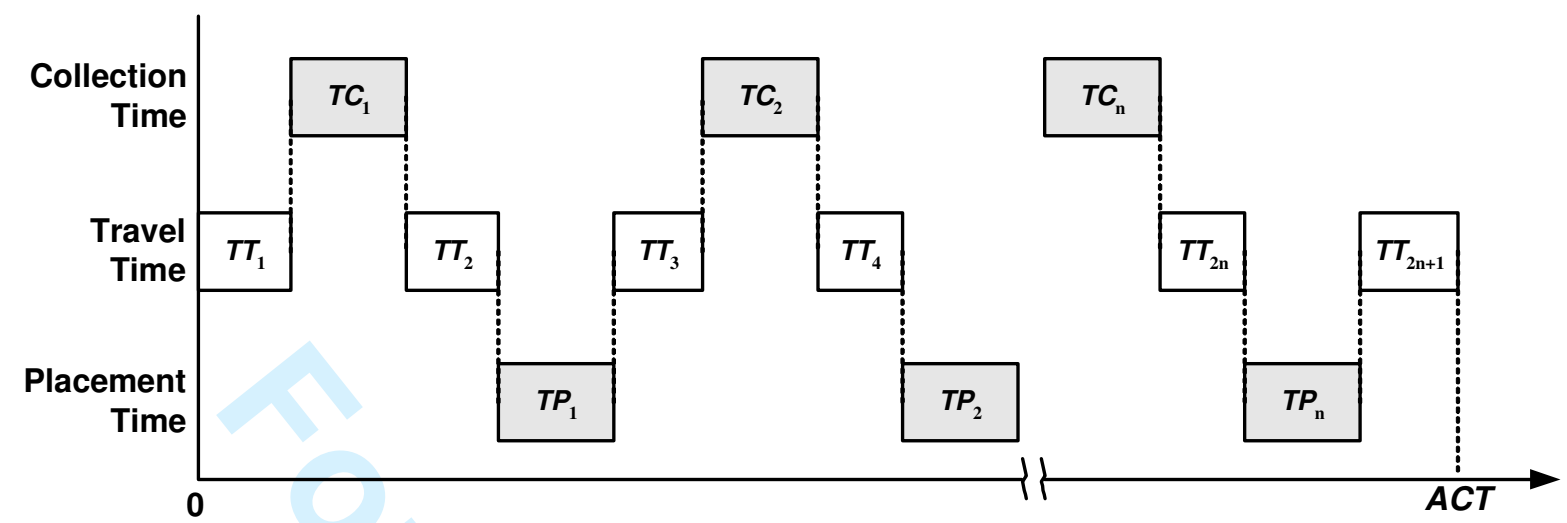

Figure 3. Assembly cycle time (ACT) 


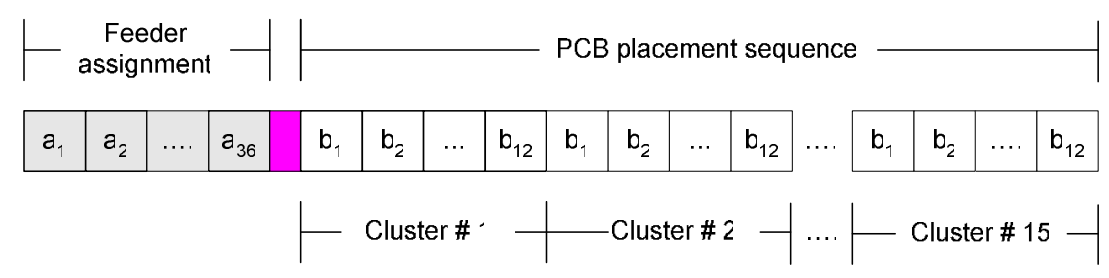

Figure 4. The chromosome structure based on the small cluster approach (SCO) 
Figure 5. The chromosome structure based on the large cluster approach (LCO) 
Figure 6. GA chromosome structure for the dual-gantry machine $\left(\mathrm{a}_{\mathrm{i}}\right.$ and $\mathrm{a}_{\mathrm{i}}$ : Component types assigned to magazine 1 and 2 , respectively, $\mathrm{i}=1,2, \ldots, \mathrm{m}$, and $\mathrm{j}=1,2, \ldots, \mathrm{p}$; $b_{i}$ and $b_{j}$ : Sequence of placement operations for head 1 and 2 , respectively, $i=1,2, \ldots, n$ and $j=1,2, . ., q$ ) 
Table 1. Characteristics of the collect-and-place machines

\begin{tabular}{lc}
\hline Velocity in $\mathrm{x}$ and $\mathrm{y}$ directions $(\mathrm{mm} / \mathrm{sec})$ & 800.0 \\
Rotational cycle time $(\mathrm{sec})$ & 0.05 \\
Placement time (sec) & 0.04 \\
Pick-up time (sec) & 0.04 \\
Feeder slot width (mm) & 8.0 \\
\hline
\end{tabular}

13

14

15

16

17

18

19

20

22

23

24

25

26

27

29

30

31

33

34

35

36

39

40

41

42

43

44

46

47

48

49

50

51 
Table 2. GA configuration used in the numerical experiments

Population size Crossover method 2000

Crossover rate

Mutation method

Mutation strategy

Mutation rate

Mutation decay ratio

Uniform-based

$90 \%$

Inversion

Parent selection method

Exponentially decreasing

$10 \%$

$50 \%$

Roulette wheel 
Table 3. Comparison of the GA approaches (single-gantry collect-and-place machine)

\begin{tabular}{|c|c|c|c|c|c|c|c|c|c|}
\hline \multirow[t]{2}{*}{$\begin{array}{l}\text { PCB } \\
\text { type* }\end{array}$} & \multicolumn{3}{|c|}{$\begin{array}{l}\text { Assembly cycle } \\
\text { time (sec) }\end{array}$} & \multicolumn{3}{|c|}{$\begin{array}{l}\text { Deviation from best } \\
\text { GA solution }(\%)\end{array}$} & \multicolumn{3}{|c|}{ CPU time (sec) } \\
\hline & $\mathrm{SCO}$ & $\mathrm{LCO}$ & $\mathrm{AO}$ & $\mathrm{SCO}$ & $\mathrm{LCO}$ & $\mathrm{AO}$ & SCO & $\mathrm{LCO}$ & $\mathrm{AO}$ \\
\hline$(120,36)$ & 31.11 & 30.63 & 30.48 & 2.07 & 0.48 & 0.00 & 39 & 155 & 236 \\
\hline$(120,60)$ & 31.53 & 30.68 & 30.63 & 2.92 & 0.17 & 0.00 & 60 & 110 & 334 \\
\hline$(240,36)$ & 61.39 & 60.46 & 60.17 & 2.02 & 0.47 & 0.00 & 156 & 782 & 2101 \\
\hline$(240,60)$ & 62.93 & 60.95 & 60.52 & 3.97 & 0.71 & 0.00 & 196 & 779 & 4289 \\
\hline$(360,36)$ & 92.16 & 90.39 & 90.60 & 1.96 & 0.00 & 0.22 & 634 & 2507 & 6341 \\
\hline$(360,60)$ & 91.87 & 89.75 & $94.05 * *$ & 2.36 & 0.00 & 4.79 & 510 & 3497 & $7200 * *$ \\
\hline
\end{tabular}


Table 4. Comparison of the LCO approach against the savings-based heuristics (single-gantry collect-and-place machine)

\begin{tabular}{cc}
\hline PCB type* & $\begin{array}{c}\text { Average improvement of } \\
\text { assembly cycle time upon } \\
\text { savings-based heuristics }(\%)\end{array}$ \\
\hline$(120,36)$ & 2.11 \\
$(120,60)$ & 3.28 \\
$(240,36)$ & 1.16 \\
$(240,60)$ & 2.32 \\
$(360,36)$ & 0.09 \\
$(360,60)$ & 0.75 \\
\hline
\end{tabular}

* (no. of components, no. of component types)

19

20

21

22

23

24

25

26

27

28

29

30

31

32

33

34

35

36

37

38

39

40

41

42

43

44

45

46

47

48

49

50

51

52

53

54

55

56

57

58

59

60

http://mc.manuscriptcentral.com/tprs Email: ijpr@lboro.ac.uk 
Table 5. Results of the single- and multi feeder assignment strategy

\begin{tabular}{ccccccc}
\hline \multirow{2}{*}{$\begin{array}{c}\text { PCB } \\
\text { type* }\end{array}$} & \multicolumn{2}{c}{$\begin{array}{c}\text { Assembly cycle time per PCB } \\
(\mathrm{sec}) * *\end{array}$} & \multicolumn{2}{c}{$\begin{array}{c}\text { No. of placement operations for } \\
\text { gantry } 1,2 * *\end{array}$} & \multicolumn{2}{c}{ Waiting time for gantry $1,2(\mathrm{sec}) * *$} \\
\cline { 2 - 7 } & $\begin{array}{c}\text { single-feeder } \\
\text { assignment }\end{array}$ & $\begin{array}{c}\text { multi-feeder } \\
\text { assignment }\end{array}$ & $\begin{array}{c}\text { single-feeder } \\
\text { assignment }\end{array}$ & $\begin{array}{c}\text { multi-feeder } \\
\text { assignment }\end{array}$ & $\begin{array}{c}\text { single-feeder } \\
\text { assignment }\end{array}$ & $\begin{array}{c}\text { multi-feeder } \\
\text { assignment }\end{array}$ \\
\hline$(120,36)$ & 17.45 & 16.22 & $54.4,65.6$ & 60,60 & $2.40,0.11$ & $1.26,0.56$ \\
$(120,60)$ & 17.25 & 16.20 & $54.4,65.6$ & 60,60 & $1.81,0.10$ & $1.29,0.50$ \\
$(240,36)$ & 32.62 & 30.64 & $114.6,125.4$ & 120,120 & $2.35,0.13$ & $1.47,0.52$ \\
$(240,60)$ & 32.70 & 30.38 & $114.6,125.4$ & 120,120 & $1.47,0.27$ & $1.34,0.68$ \\
$(360,36)$ & 48.03 & 44.83 & $178.2,181.8$ & 180,180 & $1.99,1.18$ & $1.34,1.23$ \\
$(360,60)$ & 48.05 & 44.82 & $174.0,186.0$ & 180,180 & $2.67,0.26$ & $1.40,0.75$ \\
\hline
\end{tabular}

* (no. of components, no. of component types); ** Average values for five problem instances 
International Journal of Production Research, Vol. X, No. X, 2006, xxx-xxx

O. Kulak, I.O. Yilmaz, and H.-O. Günther

PCB assembly scheduling for collect-and-place machines using genetic algorithms

\title{
PCB assembly scheduling for collect-and-place machines using genetic algorithms
}

\author{
O. KULAK†, I.O. YILMAZ + AND H.-O. GÜNTHER†* \\ $\uparrow$ Dept. of Industrial Engineering, Pamukkale University, Kinikli, 20020 Denizli, Turkey \\ $\$$ Dept. of Production Management, Technical University of Berlin, Wilmersdorfer Str. 148, 10585 Berlin, \\ Germany \\ *Corresponding author. Email: Hans-Otto.Guenther@TU-Berlin.de
}

In printed circuit board (PCB) assembly, collect-and-place machines, which use a revolver-type placement head to mount electronic components onto the board, represent one of the most popular types of assembly machinery. The assignment of feeders to slots in the component magazine and the sequencing of the placement operations are the main optimization problems for scheduling the operations of an automated placement machine. In this paper, we present different genetic algorithms (GAs) for simultaneously solving these highly interrelated problems for collect-and-place machines in PCB assembly. First we consider singlegantry machines as the basic type of machinery. In the conventional GA approach all placement operations and the feeder-slot assignment are represented by a single chromosome. In order to increase the efficiency of the genetic operators, we present a novel GA approach, which integrates a clustering algorithm for generating sub-sections of the PCB and grouping the corresponding placement operations. It is shown that the proposed GAs can be extended to schedule dual-gantry placement machines, which are equipped with two independent placement heads and two dedicated component magazines. Hence, component feeders have to be allocated 
between the two magazines. To solve this allocation problem, two different heuristic strategies are proposed. Finally, detailed numerical experiments are carried out to evaluate the performances of the proposed GAs.

Keywords: genetic algorithm; PCB assembly; collect-and-place machine; unique setup

\section{Introduction}

The assembly of electronic control units typically utilizes printed circuit boards (PCBs) to accommodate the various electronic devices. Depending on the industrial production environment, different configurations of the assembly system and different setup strategies can be used (cf. Yilmaz et al., 2006). For instance, in a mass production environment, where a small variety of PCB types are assembled in large batch sizes, the socalled unique setup represents the predominant strategy. According to this strategy, the machine is fine-tuned for the assembly of each individual PCB type in order to reduce the assembly time per board. This can be achieved by optimizing the assignment of component feeders to slots in the component magazine of the assembly machine and, at the same time, optimizing the sequence of the individual component placement operations. These optimization problems are highly interrelated and, thus, should be solved simultaneously. Specifically, genetic algorithms (GA) allow to integrate the relevant sub-problems in a joint solution procedure by use of a chromosome representation, which reflects the feeder-slot assignment as well as the operation sequence. Hence, the application of GA seems to be appropriate to support operations scheduling in PCB assembly. However, each scheduling approach, GA or conventional, has to consider the specific operation mode of the assembly machine.

In industry, a tremendous diversity of assembly machines exists. For an overview of operating principles of placement machines see Egbelu et al. (1996). According to the degree of concurrency, sequential and concurrent machines can be distinguished. The latter type of machine usually employs a beam-type placement head with a set of nozzles arranged along the robot arm in order to simultaneously pick up several components from the magazine and place them one-by-one onto the board (cf. Lee et al., 2000; Sun et al., 2005). With this type of machine, however, it is almost impossible to exploit its full capacity, because the arrangement of feeders in the component magazine and the composition of the placement locations on the board have to comply with the geometrical distances of the nozzles on the robot arm. Additionally, the number of nozzles on the beam is lower compared to the revolver-type head of a sequential placement machine. Thus, a considerably larger number of placement tours is needed. Therefore, sequential assembly machines are preferred in many industrial applications. 
For a long period of time, the most popular types of sequential assembly machines have been the pick-andplace machine with single transfer of components from the magazine to the board and the chip-shooter type of machine, which uses a rotary turret to transfer the components from the magazine to the board. Meanwhile, the chip-shooter has almost disappeared from the market and has been replaced by the so-called collect-andplace machine, which first loads a certain number of components into the revolver-type placement head and then places them sequentially onto the board. Collect-and-place machines have become popular in industry mainly because they provide a high degree of flexibility with respect to the range of component types that can be assembled and, at the same time, allow a considerable assembly speed compared to other types of machinery. Collect-and-place machines have several advantages over chip shooters. One prime advantage is that the PCB resides on a stationary table during the placement process. Thus defects are avoided, which are due to acceleration forces moving components away from their original placement position. A second advantage is the online splicing capability of collect-and-place machines, i.e. the possibility to refill component feeders without halting the machine. This paper focuses on two variants of the collect-and-place machine, namely single and dual-gantry variants of the basic machine type (see figure 1).

\section{FIGURE 1}

The general working principle of a collect-and-place machine can be described as follows. During assembly the PCB resides on the stationary work table (see figure 1). Electronic components are retrieved from the magazine, which accommodates a limited number of component feeders. Each feeder holds components of one single type. The number of slots occupied by a feeder depends on the dimensions of the components. The main characteristic of the collect-and-place machine is the design of the placement head as a rotary revolvertype unit, which is equipped with a number of nozzles, typically 6 or 12 . The placement head is located at a gantry system. In the first step of the assembly cycle, the placement head travels to the component magazine and collects components from different feeders performing stepwise rotary movements. Next, the placement head travels to the PCB and places the components one-by-one at pre-defined positions on the PCB. No reverse rotation of the placement head is allowed. Hence, the placement sequence on the PCB is always the same as the pick-up sequence from the magazine. After the placement tour is completed, the placement head travels back to the component magazine, collects the next set of components and starts a new placement tour. In order to increase the assembly speed, the dual-gantry type of collect-and-place machine has been introduced (see figure 1b). With this type of machine a collect and a placement cycle can be performed 
concurrently. While the first placement head collects components from the magazine, the other head executes the placement operations, and vice versa. For obvious reasons, interference of the operations of the two gantries has to be avoided. In the ideal case, the placement speed is doubled compared to single-gantry machines. However, this effect is barely achieved in practice.

The objective in scheduling the operations of PCB assembly machines is to minimize the assembly cycle time per board. For a collect-and-place machine, this objective is achieved by simultaneously solving the following optimization problems:

1. allocation of individual component feeders to positions in the magazine,

2. assignment of placement operations to the various tours of the placement head,

3. sequencing the placement operations within each tour and determining the travel direction of each tour, i.e. clockwise or counter-clockwise,

4. determining the sequence of the tours in an overall tour, which includes all of the necessary placement operations for a specific PCB.

In the case of dual-gantry machines, the following additional optimization problems have to be solved:

5. balancing the workload between the two gantries,

6. allocating the feeders between the two magazines.

The conventional approach to solve these highly interrelated optimization problems is to apply a multi-stage hierarchical solution procedure. As mentioned before, GA offers the advantage to simultaneously tackle the various sub-problems in one integrated approach. The main contribution of this paper is to develop different GA-based solution approaches for scheduling the operations of collect-and-place machines with revolver-type placement heads, a machine type which gains increasing popularity in industrial PCB assembly. It is shown how the basic GA approach can be adapted for the case of dual-gantry machines, for which to our best knowledge no adequate solution procedure has been published yet. In addition, we provide a numerical evaluation, which demonstrates the superiority of the GA-based approach over a conventional hierarchical solution procedure.

The remainder of this paper is organized as follows. The next section provides a brief review of the related literature. In section 3, the basic concept of the GA application in PCB assembly is introduced. First we focus on single-gantry collect-and-place machines. Different types of GA for this basic machine type are presented in section 4. Next, dual-gantry machines are considered in section 5. It is shown how the proposed GAs can be adapted for this variant of assembly machine and how the problem of allocating the component feeders 
between the two component magazines can be solved. Finally, a numerical investigation is carried out in order to evaluate the performance of the presented GAs (section 6).

\section{Literature review}

The feeder-slot assignment and the operation sequencing problem represent key issues in scheduling the operations of any PCB assembly machine. Both problems are combinatorial in nature and known to be NPhard, i.e. it is merely impossible to obtain optimal solutions to real-life problems within acceptable computational time (cf. van Laarhoven and Zijm, 1993). Typically, the approaches that have been published in the academic literature for different types of machinery rely on the divide-and-conquer principle, i.e. they apply a hierarchical procedure, in which one of the interrelated sub-problems is solved first and its solution is then used as input to the next stage of the solution procedure (e.g. van Laarhoven and Zijm, 1993; Sohn and Park, 1996; Magyar et al., 1999; Grunow et al., 2003 and Grunow et al., 2004).

Although there has been a considerable interest in analyzing different types of assembly machinery, only a few research papers have been published which focus on optimizing the operations of collect-and-place machines. For instance, Altinkemer et al. (2000) developed a model, which analyzes the feeder assignment and placement sequencing problems. Unfortunately, their approach is based on some rigid assumptions. For instance, they assume that separate tours are created for each component type and they neglect the rotational cycle time of the placement head. These principal limitations also hold for the recent paper by Kazaz and Altinkemer (2003) which expands the scope of Altinkemer et al. (2000) merely by allowing for multiple setups of the same component type. Another investigation of collect-and-place machines has been conducted by Grunow et al. (2004). In their study, they develop a three-stage heuristic approach for operations planning of a real single-gantry collect-and-place machine. Recently, dual-gantry collect-and-place machines with revolver-type placement heads have been introduced by various machine vendors. However, papers dealing with this type of machinery are lacking in the academic literature so far.

Various GA-based approaches have been developed for optimizing PCB assembly operations. For instance, Khoo and $\mathrm{Ng}$ (1998) propose a GA for the optimization of the placement sequence in semi-automated PCB assembly. However, they do not consider the feeder assignment problem and the proposed algorithm is tested only on small problem instances. Both the component placement sequence and the feeder assignment problem have been investigated later for a chip-shooter machine by Khoo and Loh (2000). Wang et al. (1999) apply GA to optimize the feeder assignment problem for a specific machine type with a single placement head and provide a comparison of GA against other optimization methods. Hong et al. (2000) developed a biological 
immune algorithm including GA principles for optimizing the operations of a multi-head surface mount placement machine. They organize the solution procedure problem into four phases, namely the assignment of component feeders to assembly heads, construction of feeder groups, feeder group assignment, and component cluster sequencing. Loh et al. (2001) propose a GA for solving the feeder assignment and component placement sequence problems of a Quad IIIC machine. Their methodology performs better compared to the GA-based approaches of Wong and Leu (1993) and Rubinovitz and Volovich (1994). However, the variety of problem instances used in the experiments is rather small: the largest PCB requires 200 components, but only 25 component feeders. Deo et al. (2002) provide a GA solution considering multiple setups for a specific PCB assembly machine which is equipped with a multi-purpose magazine allowing components to be retrieved both from tapes, which already hold the electronic components in the pre-determined placement sequence, and regular component feeders. Ho and Ji (2003) present a hybrid GA (HGA) comprising three different heuristics to solve the PCB assembly scheduling problem for a chip-shooter machine. However, they examine only one small-sized type of PCB with 10 component types and 50 operations to evaluate the HGA. Sun et al. (2005) propose a hybrid methodology including GA for optimizing the operations of a concurrent placement machine. In their approach, GA is used for solving the component allocation and feeder assignment problems in combination with a simple greedy heuristic for balancing the workload between the placement heads. However, their approach is not directly applicable to sequential placement machines, which are predominant in industrial PCB assembly.

The approaches mentioned above have some major deficits. Either the interrelated problems of feeder assignment and operations sequencing are not treated simultaneously or the developed approaches are not appropriate for PCBs of realistic size. It should be noted that, in practice, often PCBs are combined into one panel, i.e. an offset PCB consisting of a number of small-sized PCBs of the same type. This is especially useful if PCBs for small-sized final products, e.g. mobile phones, have to be produced. After the assembly of the offset PCB is completed, it is cut into individual boards for use in the assembly of the final products. Hence, a large number of placement operations per PCB, e.g. 200-400, are quite common. However, the GAbased approaches, which can be found in the literature, mainly consider PCBs of unrealistic size. An obvious disadvantage of conventional GA approaches is that their performance rapidly deteriorates for PCBs with a larger number of placement operations, because an increased number of genes in a chromosome clearly reduces the efficiency of the GA operators. To overcome this weakness, this paper presents a novel GA approach, which utilizes a more compact coding scheme and thus is also applicable to large-sized PCBs. 


\section{Application of GA to the PCB assembly problem}

In contrast to conventional search techniques, GA starts with an initial set of random solutions called population (Gen and Cheng, 1997). Each individual in the population is called a chromosome, representing a solution to the problem. Chromosomes consist of a series of elements, which are called genes. Chromosomes create new off-springs with the help of evolutions generated through a number of successful iterations. For each generation (or iteration), each chromosome in the population is evaluated by use of a fitness function. The new chromosomes, called off-springs, are created by pairing two chromosomes in the actual population using crossover operators as well as by modifying a chromosome by use of a mutation operator. A number of parent chromosomes and off-springs are selected according to their fitness values, whereas the remainder is eliminated to keep the population size constant. This way, a new generation is created. The chromosome, providing the best solution to the specific optimization problem is achieved after a number of iterations.

In the following, the key elements of the proposed GA for determining the feeder-slot assignment and the placement sequence of components on the PCB are presented.

\subsection{Structure of the chromosome}

The single-gantry collect-and-place machine is equipped with a single magazine, which holds a number of feeders, and a single placement head. The components to be assembled are collected by the placement head and attached at the corresponding positions onto the PCB. Therefore, a chromosome structure consisting of two partial links can be used to encode the PCB assembly operations (see Figure 2). The first part of the partial-link chromosome represents the feeder-slot assignment, whereas the placement sequence is coded in the second part. This chromosome structure offers the advantage of applying the GA operators to both parts of the chromosome simultaneously.

\section{FIGURE 2}

The composition of the placement tours can easily be derived from the chromosome. Let $n z$ be the number of nozzles on the placement head. So the first $n z$ genes in the right part of the chromosome indicate the sequence of placement operations in the first tour. The successive tours are derived accordingly. Only the final tour may contain less than $n z$ placement operations. Also the arrangement of genes in the chromosome implicitly determines the direction of the tour, i.e. traveling the tour clockwise or counter-clockwise, and the sequence 
of tours in the entire assembly cycle. It should be noted that in a non-GA approach, these inherent subproblems have to be solved by use of separate heuristic procedures.

In figure 2, we use the conventional approach to encode the placement operations, in which the entire sequence of placement operations is represented in the placement sequence part of the chromosome. However, a computationally more efficient chromosome structure is achieved by clustering placement operations into tours of the placement head and by applying genetic operators to these compact chromosome sections. This advanced approach is presented in section 4.

\subsection{Creation of the initial population}

GA starts searching with a large randomly generated chromosome set, which is called the initial population. Generation of chromosomes with a very good fitness value is quite difficult due to randomness. In the proposed methodology, the half of the initial population delivering the better fitness values is selected as the GA population.

\subsection{Fitness function}

In each generation all chromosomes in the population are evaluated by use of the fitness function. The chromosomes with better fitness values are included in the mating pool to form the new off-springs. Hence, selection of chromosomes with better fitness values enables the GA to reach good solutions in a shorter period of time. The fitness function of the proposed GA is defined as the assembly cycle time $(A C T)$ per PCB, which is illustrated in figure 3. For this purpose, the placement sequence coded in the second part of the chromosome is converted into tours of the placement head and the placement time $(T P)$ needed for the given sequence is calculated. At the same time, the feeder assignment part of each chromosome provides information about the pick-up locations of the components, which have to be collected from the magazine before the placement tour starts. This time is defined as the collection time (TC). It should be noted that components are always placed on the board in the same order as they are picked up from the magazine. Travel times $(T T)$ of the placement head during the collection and the placement phase as well as the time needed to travel from or back to the parking location (at the beginning and end of each assembly cycle) are also integrated into the fitness function value. Since the placement head moves simultaneously in both directions, the Tschebyscheff distance measure is used to calculate the travel distances of the placement head. (Note that, according to the Tschebyscheff distance measure, the maximum of the travel times in the $\mathrm{x}-$ and the $\mathrm{y}$ direction determines the effective travel time.) The rotational cycle time of the placement head is considered 
as the minimum time between two successive picking or placement operations, because a move to the next index position of the revolver must always take place before the next component is picked up or placed. The chromosome offering the smallest assembly cycle time is taken as the best current solution.

\section{FIGURE 3}

\subsection{Parent selection methods}

In GA, one or more off-springs are generated from the chromosomes, which are selected for the mating process. The defined process cannot begin before a mating pool is created for each generation. The selection of the chromosomes from the current population to be included in the mating pool is important for the performance of the GA. In this study, the chromosomes, which show an equal or better fitness value than the average, are included in the mating pool. The selection of the chromosome parents for generating new offsprings is carried out by using the roulette wheel, rank weighting and tournament methods, respectively.

\subsection{GA operators}

In order to create new off-spring chromosomes with better fitness values, different crossover and mutation operators are applied.

Crossover operators: Crossover operators are used to find better off-springs by combining the characteristics of two parent chromosomes. In the literature, a variety of crossover operators can be found. In this study, four crossover operators are implemented and tested, namely the order crossover (OX), the position-based (uniform-based) crossover, the partially mapped crossover (PMX) and the cycle crossover (CX). An overview of these crossover operators can be found in Gen and Cheng (1997).

Mutation: Mutation is an essential GA operator, which allows the GA to enter different sections of the solution space during the search process. Thus, the acceptance of a local optimum as the best solution can be avoided with the help of this operator. The proposed methodology includes swap, inversion, left and right rotation and mixed mutation operators. The latter operator selects one of the given four mutation methods randomly and applies it to the observed generations. Hence, different mutation operators are used for different generations. Another important element affecting the performance of the mutation operator is the definition of the mutation rate. For GA applications, the use of a fixed mutation rate for all generations is quite common. Nevertheless, an exponentially decreasing mutation rate is also integrated into the proposed GA methodology. 


\subsection{Termination condition}

Two conditions are considered simultaneously for terminating the GA iterations, namely the computational time and the convergence of the fitness value. If both the average and the best fitness values of the current population are equal to the corresponding values of the next generation for a given number of successive iterations, the search process is terminated. In addition, a CPU time limit is defined, in order to restrict the computational effort.

\section{GA approaches for single-gantry collect-and-place machines}

According to the research results reported in the academic literature, the performance of GA declines when the number of assembly operations and component feeders increases. This is due to the growing number of genes in the chromosome. As a result, the efficiency of the crossover operators is impaired. In order to improve the performance of the GA and to overcome this deficiency, several approaches based on alternative chromosome structures are presented in the following.

\subsection{Consideration of all operations in a single set (AO)}

The chromosome structure (denoted here by AO, i.e. "all-in-one"), which has been commonly used in the literature, considers all placement operations as a single unstructured set, i.e. no grouping of placement operations for adjacent placement locations takes place. Hence, the number of component types to be assembled determines the number of genes in the feeder assignment part of the chromosome and the number of placement operations determines the length of the placement sequence part. This chromosome structure is illustrated in figure 2 above.

\subsection{Consideration of small clusters of operations (SCO)}

In order to circumvent the weakness of the traditional GA, which considers all operations in a single unstructured set, and to improve the computational efficiency, modifications of the conventional GA approach are proposed. One of these modifications is based on the definition of small clusters of operations (SCO). In this approach the entire set of placement operations is sub-divided into sub-sets of operations (usually 6 or 12) according to the number of nozzles on the placement head of the assembly machine. Hence, these small clusters represent actual assembly tours. In our approach, the construction of clusters is carried out by utilizing the density search construction method (DSCM) of Ahmadi and Osman (2005). This clustering 
technique has been chosen because it explicitly takes limitations on the cluster size into account and it has shown to be very competitive against other types of clustering algorithms. DSCM selects some positions as cluster centers depending on the density concept and assigns the remaining positions to these centers considering regret values with respect to each center.

We define the following notation.

$n z: \quad$ Number of nozzles on the placement head (maximum cluster size)

$l_{i}$ : $\quad$ Location of placement operation $i$ on the PCB

$L_{i}$ : $\quad$ Set of $(n z-1)$ nearest locations of $l_{i}$

$T_{i}$ : $\quad$ Sum of distances from $l_{i}$ to all locations in $L_{i}$

$D_{i}$ : $\quad$ Density value for $I_{i}\left(=n z / T_{i}\right)$

$P L: \quad$ List of placement operations

The entire clustering procedure can be summarized as follows.

Step 1. Compute the $D_{i}$ values for all placement locations.

Step 2. Sort the $D_{i}$ values in decreasing order in list $P L$.

Step 3. Select the location $l_{i}$ at the top of $P L$ as the temporary center.

Step 4. Assign the locations in $L_{i}$ to $l_{i}$ in order to generate a cluster.

Step 5. Determine the center for the generated cluster. The center of cluster $L_{i}$ is defined as the location with the smallest $T_{i}$ value among all $I_{i}$ in $L_{i}$. (Note that $T_{i}$ values are calculated using the Tschebyscheff distance measure.)

Step 6. Repeat steps 3-5 until all locations are assigned to a center. (Note that the last cluster may include less than $n z$ placement locations depending on the total number of placement operations.)

Step 7. Reassign all placement locations to their nearest center in decreasing order of their regret values. The regret value of $l_{i}$ is defined as the difference between the distances from $I_{i}$ to its nearest and second-nearest centers determined in step 5.

After the clusters are generated by use of the DSCM procedure, the resulting centers of the clusters are sorted in ascending order of their $x$-coordinates. For each tour, the movement time of the placement head from the final pick-up location at the magazine to the location of the first placement operation of the corresponding cluster is mostly dominated by the travel time in the $y$-direction. (Note that Tschebyscheff distance measures are used.) Hence, the $y$-distances have to be traveled for each cluster in any case. Therefore, it seems reasonable to neglect the sequence of the clusters in this approach. Thus, we assign the clusters in ascending 
order of the $x$-coordinates of their center to the genes in the chromosome. As an example, figure 4 illustrates the chromosome structure based on the small cluster approach for a PCB with 36 component types and 180 placement operations. As the maximum number of placement operations per cluster has been set at 12, a total of 15 clusters is constructed each comprising 12 placement operations. During the creation of the initial population, random chromosomes are generated by assigning each placement operation to a random position in the corresponding cluster. The genetic operations are then applied to each cluster separately to determine the sequence of operations within a tour.

\section{FIGURE 4}

\subsection{Consideration of large clusters of operations (LCO)}

This methodology combines the strengths of both the AO and the SCO approaches. Using AO the coding of the placement operations is more detailed and thus the search space is broader. In contrast, the SCO approach allows more efficient use of the genetic operators, but its performance depends heavily on the solution to the clustering problem. The idea behind the LCO approach is to generate larger clusters of placement operations in order to further increase the computational efficiency of the GA. As an example, consider a PCB which has to be populated with 180 components. A feasible large cluster definition could be based on a number of 60 placement operations per cluster. As a result, three large clusters, each comprising 60 placement operations, are created. The resulting chromosome structure is shown in figure 5.

\section{FIGURE 5}

A key issue of the LCO approach is the determination of the cluster size. We observed the best performance and GA operator efficiency for a cluster size in the range of $[60,96]$, where the lower and upper limits represent integer multiples of the number of nozzles (typically 6 or 12) on the placement head. Based on this observation, a more general procedure to determine the size of the large clusters can be described as follows.

Step 1. Set the candidate number of clusters to 1.

Step 2. Divide the number of placement operations by the candidate number of clusters and calculate the cluster size, i.e. the number of placement operations in each cluster. 
Step 3. Increment the candidate number of clusters by one and repeat step 2 until the cluster size is less than the pre-defined lower limit for cluster size.

Step 4. Only the numbers of clusters resulting in cluster sizes within the pre-defined range of cluster sizes are considered further.

Step 5. Determine the number of clusters which results in the smallest remainder to any of the pre-defined cluster sizes. The corresponding cluster size is chosen as the number of genes in a large cluster.

An elementary example shall be used to illustrate this procedure. Consider a PCB with 250 placement operations and pre-defined cluster sizes of, 60,72, 84, and 96. (Note that 12 is assumed here as the number of nozzles on the placement head.) In the first three steps of the procedure, the following cluster sizes are determined: $250 / 1=250,250 / 2=125,250 / 3=83.33$, and $250 / 4=62.5$. After step 4 , only denominators (numbers of clusters) of 3 and 4 are considered further, since these denominators give cluster sizes in the feasible range of 60 to 96 genes. These denominators show remainders of 0.67 (to cluster size of 84) and 2.5 (to cluster size of 60), respectively. Thus, 84 is chosen as the cluster size in step 5 of the procedure. Finally, the 250 placement operations are allocated to two clusters of size 84 and one cluster of size 82 .

Similar to the SCO approach, small clusters must again be determined by applying the DSCM procedure as explained in section 4.2. Next, the corresponding small clusters are sorted in ascending order of the $x$ coordinates of the cluster centers. Accordingly, small clusters are assigned one-by-one until the number of genes defined for a large cluster size is reached. During the creation of the initial population, genes are generated randomly from the components assigned to each large cluster. As in the SCO approach, genetic operators are separately applied to each cluster.

It should be noted that the LCO approach only makes sense for large-sized PCBs, i.e. boards which require considerably more than 60 placement operations. Otherwise, the AO or the SCO approach should be used.

\section{GA approaches for dual-gantry collect-and-place machines}

This section extends the basic GA approaches to the case of a dual-gantry collect-and-place machine. As mentioned before, this type of machine is equipped with two separate placement heads and two dedicated component magazines and thus offers the advantage to synchronize the collection and placement phases of the two placement heads. This is the main reason, why the dual-gantry variant of the machine has gained widespread use in industry. Since each of the component magazines is dedicated to a specific gantry, all component feeders must be assigned to one of the magazines. To solve this allocation problem, two different 
strategies are proposed in section 5.1. One is based on the assignment of feeders to one single gantry (section 5.1.1). The other permits the duplication of feeders and thus the assignment of component types to both gantries (section 5.1.2). The extended GA approach for dual-gantry machines is then laid out in section 5.2.

\subsection{Feeder allocation}

\subsubsection{Single-feeder assignment}

The main objective of the feeder allocation procedures is to assign component feeders to the magazines of the placement machine such that the workload of both gantries is balanced. In the ideal case, each gantry carries out an equal number of placement tours and the time required to complete the collection and placement phase of each pair of corresponding tours are the same for both gantries.

To solve the component allocation problem, it seems reasonable to define the workload of a gantry as the number of placement operations assigned to it. In the single-assignment strategy, it is assumed that each component type can only be assigned to one of the gantries (component magazines). This involves the assignment of all placement operations of a specific component type to the selected gantry.

The proposed heuristic procedure for the assignment of component types (feeders) to one of the gantries consists of the following steps.

Step 1. Determine the usage of each component type as the total number of corresponding placement operations. Sort the component types in descending order of their usage.

Step 2. For each component type determine the "closer" gantry in the $y$-direction, i.e. the one that is the nearest for the majority of the corresponding placement locations. (For example, if two copies of a specific component type have to be placed nearer to gantry (magazine) 1 and one copy nearer to gantry (magazine) 2 , then gantry 1 is considered as "closer".)

Step 3. Assign the first component type in the list of step 1 to the "closer" gantry (component magazine).

Step 4. If the workload difference between the two gantries is smaller than the number of nozzles on the placement head, i.e. the maximum number of placement operations per tour, assign the next component type in the list of step 1 to the "closer" gantry. Else, assign it to the gantry, where the workload is lower.

Step 5. Repeat step 4 until all component types are assigned to either of the gantries. 
Step 6. In the case of an unequal number of tours at the two gantries, reassign feeders from the overloaded gantry to the opposite one, until the absolute difference between the number of tours of both gantries is less than or equal to one.

\subsubsection{Multi-feeder assignment}

In the single-feeder assignment strategy, all placement operations of a component type are carried out by the selected gantry. Thus, balancing the workload between the two gantries can become a very tough task, if the component types greatly differ in the number of corresponding placement operations. Hence, duplicating a component feeder and assigning the component type to both gantries and thus re-allocating part of the placement operations to the opposite gantry can be advantageous in order to balance the workload. The disadvantage of feeder duplication can be seen in the increased capital investment in both the feeder equipment as well as in the additional supply of electronic components. This economical evaluation, however, is beyond the scope of this paper. Yet, in many practical applications a limited number of feeders for highusage components is often set up more than once on a machine or an assembly line.

The intention of the multi-feeder assignment strategy is to improve the workload balance between both gantries. Hence, clusters of placement operations are generated using the DSCM procedure described in section 4.2. Recall that the number of placement operations in a cluster is limited by the number of nozzles on the placement head. The clusters and the corresponding placement operations are then assigned to the two gantries by use of the following procedure.

Step 1. Create small clusters of operations according to the DSCM procedure.

Step 2. For each cluster center, determine the "closer" gantry in the $y$-direction and assign the cluster to this gantry.

Step 3. If there is an uneven number of clusters assigned to the gantries, reassign the cluster whose center is located the farthest in the $y$-direction from the gantry with the higher workload to the opposite one.

Step 4. Repeat step 3 until the absolute difference between the number of clusters assigned to the two gantries is less than or equal to one.

Step 5. For each gantry, allocate the component feeders needed to carry out the placement operations in the assigned cluster of placement operations. 


\subsection{GA chromosome structure for the dual-gantry machine}

The chromosome structure of the GA approaches for single-gantry machines is slightly modified in order to cover the specific issues of dual-gantry collect-and-place placement machines. The modified partial-link chromosome is illustrated in figure 6. After the feeder allocation has been completed by use of one of the strategies developed in the previous section, the feeder-slot assignment and the placement sequence have to be determined for each gantry. Hence, the same GA operators as defined in sections 3 and 4 are applied to the dual-gantry chromosome structure.

\section{FIGURE 6}

The fitness function value for each chromosome is defined as the total assembly cycle time. In contrast to the single-gantry machine, operations are carried out simultaneously by the two gantries. During the placement phase of the first gantry, the second gantry collects components needed for the next tour from the magazine. In the next cycle, the placement head at the second gantry places the loaded components onto the PCB, while the first gantry returns to its magazine to collect components for its subsequent tour. Hence, the procedure for calculating the assembly cycle time has to be modified accordingly.

\section{Numerical investigation}

In the following sections, the results of our numerical experiments on the proposed GA approaches for both single and dual-gantry collect-and-place machines are presented. The main research questions addressed in our numerical investigation are:

- How do the proposed GA approaches perform against each other (section 6.2)?

- How do the proposed GA approaches perform against the savings-based heuristics of Grunow et al. (2004) (section 6.3)?

- How do the proposed GA approaches and feeder allocation strategies perform for dual-gantry placement machines (section 6.4)?

\subsection{Experimental design}

The numerical experiments are conducted using a common PC with Pentium IV $3.2 \mathrm{GHz}$ processor and $1 \mathrm{~GB}$ random access memory (RAM). The presented GA approaches are coded in the Visual C++.NET 
programming language. Table 1 illustrates the main characteristics of the investigated collect-and-place machines equipped with single or dual 12-nozzle rotary placement head(s).

\section{TABLE 1}

In our numerical experiments, the total number of components per PCB has been set at 120, 240, and 360, whereas the number of component types per PCB was set at 36 and 60. Five problem instances were generated for each of the given six PCB types resulting in a total of 30 problem instances. The dimensions of each PCB are assumed as $300 \times 300 \mathrm{~mm}$. The number of components to be assembled from each component type as well as the coordinates of the placement locations have been generated randomly according to a uniform distribution.

A number of preliminary tests have been carried out to investigate the effectiveness of the GA operators defined in section 3 and to find the best GA configuration for our application. We experienced that the GA control parameters given in table 2 perform best for most of the instances. Therefore, they were applied throughout our numerical experiments. Each run of the GA was terminated when one of the following termination conditions was reached: a CPU time limit of two hours or 50 successive iterations with nondecreasing fitness values. 10 replications are executed for each GA experiment.

\section{TABLE 2}

\subsection{Comparison of the proposed $G A$ approaches}

In the first series of experiments, the performance of the GAs presented in section 4 is investigated for the case of a single-gantry collect-and-place machine. A total of six problem instances (one instance per each PCB type) were randomly generated according to the experimental design indicated above. For each problem instance, the GAs were run 10 times with different randomly generated initial populations. The best assembly cycle time per PCB out of the ten replications achieved for each GA approach and PCB type are displayed in table 3 .

\section{TABLE 3}


AO, which represents the conventional GA approach for the PCB assembly problem, performs well for all PCB types except for the most complex PCB types with 360 placement operations. However, the computational time needed is extremely high compared to the other approaches. Especially, for the PCB type $(360,60)$ no sufficient convergence was reached within two hours of CPU time. Obviously, the performance of the AO approach deteriorates with an increasing number of genes in the chromosome and the resulting enhanced solution space.

On the other hand, SCO provides very fast solutions, but its performance highly depends on the efficiency of the clustering procedure outlined in section 4.2. As the elements of each cluster are determined in advance, the solution achieved by the GA indicates only the sequence of the placement operations within each placement tour and the assignment of the component feeders to the slots in the magazine. Thus, the computational effort is considerably reduced.

In contrast to $\mathrm{AO}$ and $\mathrm{SCO}$, the LCO approach provides very good results in a relatively short computational time. The assembly cycle times achieved do not deviate by more than $0.71 \%$ from the best solution found by any of the GAs. In particular, for the large-sized PCB types with 360 placement operations the LCO approach outperforms its counterparts. The competitiveness of LCO against AO relies on the comparatively limited search area, whereas the formation of larger clusters including a number of placement tours allows LCO to generate better tour structures than the SCO approach.

\subsection{Comparison of the $G A$ approach against the savings-based heuristics}

LCO appears to be the most attractive GA approach in terms of solution quality and computational efficiency as can be seen from the numerical results presented in table 3. Therefore, in the second series of experiments, the performance of the LCO approach is compared with the results achieved by the savings-based heuristics of Grunow et al. (2004). Their approach exploits the similarity between the vehicle routing problem and the component placement problem, when collect-and-place machines are employed. Specifically, they propose four variants of the well-known savings heuristics to determine the component placement sequence. So far the heuristics of Grunow et al. (2004) are the only published algorithms for scheduling the assembly operations of a real single-gantry collect-and-place machine, e.g. the SIPLACE series from Siemens. Hence, both for the savings-based heuristics of Grunow et al. (2004) and for the LCO approach, the best results out of 10 replications for each experiment are taken. The experiments are carried out for five instances of each PCB type to be assembled by use of a single-gantry collect-and-place machine. The average improvement in 
assembly cycle time per PCB obtained by the LCO approach upon the savings-based heuristics is given in table 4 .

\section{TABLE 4}

As can be seen from table 4, the LCO approach achieves better solutions for all of the conducted experiments. The proposed GA approach tackles the feeder assignment and the placement sequencing problems simultaneously, whereas the savings-based heuristics of Grunow et al. (2004) relies on a hierarchical solution procedure, which solves the feeder assignment and placement sequence problems sequentially and, finally, applies local search techniques to improve the overall solution. Apparently, the superior performance of the GA is achieved by simultaneously solving the interrelated sub-problems of PCB assembly scheduling. Another important result of our experiments is that the improvement achieved by LCO is larger for PCBs types with a small number of placement operations. The density of the PCBs with 120 placement operations lies at 0.13 placement positions per $\mathrm{cm}^{2}$, whereas this value reaches 0.40 for PCBs with 360 placement operations. As described in section 3.3, the rotational cycle time of the revolver-type placement head has to be considered as the minimum travel time between two successive placement or pick-up positions. Thus, the number of successive positions, which can be reached within the minimum travel time, increases for PCBs with higher density. As a result, the total assembly cycle time is less sensitive to changes in the sequence of placement operations within an assembly tour. Hence, the declining improvement of the GA with the increasing number of placement operations per PCB is primarily caused by the described density effect rather than by the performance of the GA operators.

\subsection{Comparison of $G A$ approaches for the dual-gantry placement machine}

In the final series of experiments, LCO as the best performing GA approach is applied to the dual-gantry collect-and-place machine. Again, five instances per PCB type are investigated and for each instance the best result out of 10 replications of the GA is evaluated. The results of the single- and multi-feeder assignment strategies are presented in table 5.

\section{TABLE 5}


Clearly, the maximum performance of the dual-gantry collect-and-place machine can only be achieved, if the workload between the two gantries is perfectly balanced. As an indicator of workload balance, the difference between the numbers of placement operations per gantry can be considered. This measure, however, is only approximate since the time required to perform a placement operation depends on the travel time of the placement head between two adjacent operations within a tour. As a more accurate measure, the waiting time of the gantries can be considered, i.e. the time a gantry has to wait for starting its placement operations, because the other gantry has not completed its placement tour.

The numerical results given in table 5 show that the multi-feeder assignment strategy balances the workload of the gantries (number of placement operations) more effectively than the single-feeder assignment strategy. Hence, the maximum waiting time of the gantries is decreased and the assembly cycle time is reduced by $6.6 \%$ on average. This improvement, however, is realized at the expense of additional component feeders. In the ideal case, the dual-gantry machine places components twice as fast as the single-gantry collect-andplace machine. In our experiments, we observed that the dual-gantry machine achieved an actual placement speed being 1.83 and 1.96 times faster compared to the single-gantry variant, when using the single and multifeeder assignment strategy, respectively.

\section{Concluding remarks}

In this paper, three different GA approaches are proposed for scheduling the operations of collect-and-place machines in PCB assembly. We consider the case of single and dual-gantry placement machines, which despite their widespread application in industry have not been investigated extensively. While most algorithms for scheduling assembly machine operations rely on a hierarchical principle, GA offers the advantage to cover the interrelated sub-problems of feeder-slot assignment and placement sequencing in one integrated approach. Because of the mathematical complexity of the entire scheduling problem, it is practically impossible to determine optimal solutions for PCBs of realistic size. Hence, heuristics and numerical search methods have to be applied. In our numerical experiments, GA has shown to be very efficient in terms of computational time, especially if adequate coding schemes are used. Our numerical experiments revealed that the proposed GAs outperform the heuristic solution procedure of Grunow et al. (2004), which is the only efficient heuristic for single-gantry collect-and-place machines published in the academic literature.

Presently, there is an ongoing trend in industry to replace machines of the pick-and place and chip-shooter type by collect-and-place and modular placement machines. Even machines equipped with four gantries are 
being offered by several machine vendors. Hence, issues of scheduling operations for highly flexible assembly systems, which integrate multiple assembly heads equipped with interchangeable nozzles for placing specific types of electronic components, are of major importance for future research.

\section{References}

Ahmadi, S. and Osman, I.H., Greedy Random Adaptive Memory Programming Search for the Capacitated Clustering Problem. European Journal of Operational Research, 2005, 162, 30-44. Altinkemer, K., Kazaz, B., Köksalan, M. and Moskowitz, H., Optimization of Printed Circuit Board Manufacturing: Integrated Modeling and Algorithms. European Journal of Operations Research, 2000, 124, 409-421.

Deo, S., Javadpour, R. and Knapp, G.M., Multiple Setup PCB Assembly Planning Using Genetic Algorithms. Computers \& Industrial Engineering, 2002, 42, 1-16.

Egbelu, P.J., Wu, C.-T. and Pilgaonkar, R., Robotic assembly of printing circuit boards with component feeder location consideration. Production Planning and Control, 1996, 7, 162-175. Gen, M. and Cheng, R., Genetic Algorithms and Engineering Design. 1997 (Wiley: New York). Grunow, M., Günther, H.-O. and Schleusener, M., Component allocation for printed circuit board assembly using modular placement machines. International Journal of Production Research, 2003, 41, 1311-1331.

Grunow, M., Günther, H.-O., Schleusener, M. and Yilmaz, I.O., Operations planning for collect-andplace machines in PCB assembly. Computers \& Industrial Engineering, 2004, 47, 409-429.

Ho, W. and Ji, P., Component Scheduling for Chip Shooter Machines: A Hybrid Genetic Algorithm Approach. Computers \& Operations Research, 2003, 30, 2175-2189.

Hong, J., Lee, W., Lee, S., Lee, B. and Lee, Y., An Efficient Production Planning Algorithm for MultiHead Surface Mounting Machines Using the Biological Immune Algorithm. International Journal of Fuzzy Systems, 2000, 2 (1), 45-53.

Kazaz, B. and Altinkemer, K., Optimization of multi-feeder (depot) printed circuit board manufacturing with error guarantees. European Journal of Operational Research, 2003, 150, 370-394.

Khoo, L.P. and Loh, K.M., A Genetic Algorithms Enhanced Planning System for Surface Mount PCB Assembly. The International Journal of Advanced Manufacturing Technology, 2000, 16, 289-296. 
Khoo, L.P. and Ng, T.K., A Genetic Algorithm-based Planning System for PCB Component Placement. International Journal of Production Economics, 1998, 54, 321-332.

Lee, W., Lee, S., Lee, B. and Lee, Y., An Efficient Planning Algorithm for Multi-head Surface Mounting Machines Using a Genetic Algorithm. Journal of Universal Computer Science, 2000, 5, 833-854.

Loh, T.S., Bukkapatnam, S.T.S., Medeiros, D. and Kwon, H., A Genetic Algorithm for Sequential Part Assignment for PCB Assembly. Computers\& Industrial Engineering, 2001, 40, 293-307. Magyar, G., Johnsson, M. and Nevalainen, O., On solving single machine optimization problems in electronics assembly. Journal of Electronics Manufacturing, 1999, 9, 249-267.

Rubinovitz, J. and Volovich, D., Genetic Algorithm for the Robotic Assembly Plan Problem. SME Technical Paper MS940200, 1994.

Sohn, J. and Park, S., Efficient operation of a surface mounting machine with a multihead turret. International Journal of Production Research, 1886, 34, 1131-1143.

Sun, D.S., Lee, T.E. and Kim, K.H., Component Allocation and Feeder Arrangement for A Dualgantry Multi-head Surface Mounting Placement Tool. International Journal of Production Economics, 2005, 95, 245-264.

Van Laarhoven, P.J.M. and Zijm, W.H.M., Production preparation and numerical control in PCB assembly. International Journal of Flexible Manufacturing Systems, 1993, 5, 187-207.

Wang, W., Nelson, P.C. and Tirpak, T.M., Optimization of High-Speed Multi-station SMT Placement Machines using Evolutionary Algorithms. IEEE Transactions on Electronics Packaging Manufacturing, 1999, 22 (2), 137-146.

Wong, H. and Leu, M.C., Adaptive Genetic Algorithm for Optimal PCB Assembly Planning. CIRP Annals, 1993, 42 (1), 17-20.

Yilmaz, I.O., Grunow, M., Günther, H.-O. and Yapan, C., Development of group setup strategies for makespan minimization in PCB assembly. International Journal of Production Research, 2006 (to appear). 